\title{
Infusing Free, Hands-On, Interactive Information Technology Into The MIS Curriculum
}

\author{
James R. Otto, (E-mail: jotto@towson.edu), Towson University
}

\begin{abstract}
This paper provides practical suggestions for incorporating free, hands-on interactive information technology (IT) into a business Management Information Systems (MIS) course. This paper focuses on interactive multimedia technologies (such as programs, audio, video, animation, etc.) that can be accessed and used for free (subject to any software license terms). This paper provides specific details about where to access the technology, how it fits into the curriculum and any issues related to installing or using the technology.
\end{abstract}

\subsection{Introduction}

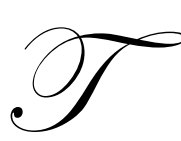

his paper provides practical suggestions for incorporating hands-on interactive information technology (IT) into a business Management Information Systems (MIS) course. Provided a PC that has a Web browser and internet connection, students can access a huge variety of exciting, interactive, and relevant learning material that is directly related to what they are learning from the textbook and instructor lectures. While the amount of information on the Web is staggering and in many forms, this paper focuses on interactive multimedia technologies (such as programs, audio, video, animation, etc.) that can be accessed and used for free (subject to any software license terms). Given the tight budgets of many colleges and universities, the no-cost aspect of these technologies can be a significant benefit. This paper provides specific details about where to access the technology, how it fits into the curriculum, and any issues related to installing or using the technology.

The information in this paper is specifically tailored for an introductory MIS course for business students, although much of the technology suggestions could probably be applied to other courses. For example, a demonstration of Internet telephony may also be appropriate for an E-business or Telecommunications course in addition to an introductory MIS course.

The general MIS course is typically a required core course that provides a broad overview of information systems from a business perspective. For example, a university catalog describes their general business MIS course as addressing:

Strategic, tactical, and operational applications of information technology including management information systems, decision support systems, intelligent systems, strategic information systems, and electronic commerce. Topics include data and knowledge management and networking computing (Towson University, 2003).

Because the course is required of all students, a wide variety of IT knowledge and skill levels are typically encountered; from MIS and computer science majors to students who know very little about computers. Hands-on, active learning techniques can be more motivating for students, help students learn, and increase their confidence with course material, and better understand what they are studying (Harton et.al., 2002). 
Based on over 6 years of teaching both undergraduate and graduate introductory MIS courses, as well as numerous other MIS courses, the author has collected a wide variety of hands-on, free IT resources available to support MIS learning. These resources include software, online demonstrations and animations, video, audio, and native operating system capabilities.

\subsection{Computer Information Systems Active Learning}

The typical introductory MIS course covers a wide variety of topics, mostly at an overview level. For example, a general introductory MIS textbook (Haag et.al, 2003) includes chapters on:

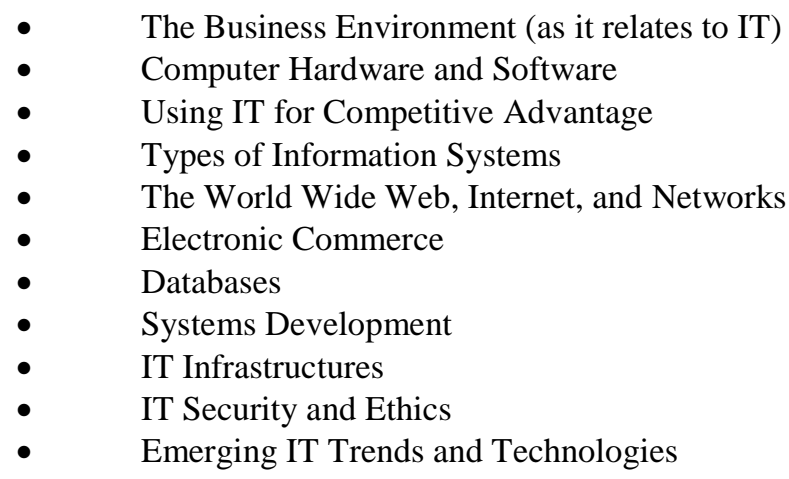

Active learning resources discussed in this paper are organized around the general themes listed above. For each IT resource, the following information is provided where appropriate: the name of the technology, where it can be found, a description of the resource, how it can be used to supplement the curriculum, and any issues related to the resource.

Certain hardware, software, and administrative resources are required to leverage these IT resources. First, students will need access to a Windows-based PC with a late model Internet Explorer Web browser (e.g., Internet Explorer 6.0+) and access to the Internet in a computer classroom or laboratory. Other Web browsers and operating systems may work, but this paper only addresses Internet Explorer on the Windows operating system. Second, many of the software applications or Web browser plug-ins (such as Macromedia Flash for flash animations) may require the installation of applications. This will probably necessitate close coordination with the system administrator to install any applications. In many cases, it may make sense from administrative and security perspectives to implement a dual boot capability. In this way, any MIS-specific applications are segregated from the general operating environment to reduce administrative headaches and potential security problems.

\subsection{Free, Interactive Information Technology Resources}

The following paragraphs list a variety of free, interactive IT resources (organized by categories) that are available to augment an MIS class.

\subsection{General Information}

Current Events Audio: Students can listen to archived current events and news stories via streaming audio at a number of online radio sites. Of particular note is http://www.npr.org, which allows one to listen to archived segments from Morning Edition, All Things Considered, Day to Day, Talk of the Nation, Fresh Air, The Tavis Smiley Show, Weekend Edition Saturday, Weekend Edition Sunday, or the Motley Fool Radio Show. A streaming audio player will be required (such as the Windows Media Player from http://www.microsoft.com or the RealPlayer from http://www.real.com), along with PC speakers.

Current Events Video: A streaming video news source is available at http://www.cbsnews.com. This site also includes stories on technology and science, as well as news on other topics (such as politics and health). A 
streaming video player (such as the RealPlayer or Microsoft Media Player) is required to play the videos. Computer speakers are also needed to listen to the audio.

\subsection{It Business Environment Resources}

Web Translation: The automated translation of sentences between a variety of languages to include English, Dutch, French, Japanese, Korean, and Chinese can be demonstrated at http://www.systranbox.com/systran/box. A sentence typed in one text box is translated and appears in a second text box.

\subsection{Computer Hardware And Software}

Computer Hardware Customization: A computer can be custom built and priced at http://www.dell.com by selecting major PC components and features to include processor speed, operating system, RAM size, hard drive specifications, CD-ROMs, DVDs, modems, monitors, and speakers.

\subsection{Types Of Information Systems}

Personal Productivity Software: Open source spreadsheet, presentation, word processing, drawing, and database software that are compatible with Microsoft Office products can be downloaded from http://www.openoffice.com. Additionally, Microsoft (http://www.microsoft.com) provides free converters and/or viewers for their Office (PowerPoint, Excel, Outlook, Project, and Visio) applications. The open source applications help faculty and students share Microsoft Office files with people who may not have Office programs or may have different versions of Office programs.

Expert System Application Demonstration: Students can work demonstration expert systems on the Web at http://www.myacquire.com/member_sites.asp.

Neural Network Application Demonstration: A neural network hand writing recognition application on the Web is available at http://members.aol.com/Trane64/java/JRec.html. The application requires that the browser run Java applets.

Genetic algorithm Application Demonstration: A genetic algorithm Web application to solve the traveling salesman problem is available at http://users.ox.ac.uk/ quee0818/ts/ts.html.

Collaboration Systems: Collaboration tools allow users to share files, conduct Web meetings, communicate, and share calendars. A flash demonstration on the Web that describes the functionality of peer-to-peer collaboration is available at http://www.groove.net/default.cfm?pagename=Workspace\#. A similar demonstration for a Web centric collaboration tool is available at https://dailydemos.webex.com/dailydemos/mywebex/default.php. A sound card is required to hear the audio portion of the demonstrations.

Customer Relationship Management (CRM) Systems: A Web flash demonstration of a CRM system is available at http://www.bbfmcreative.com/clients/8069/docs/demos/icon/default.htm. The animation involves audio and includes advertising for a Microsoft CRM product.

Supply Chain Management (SCM) Systems: Several Web flash demonstrations of SCM systems are available at http://www.navision.com/sea/view.asp?documentid=1468\&categoryid=495. The animation involves audio and includes advertising for the respective companies offering SCM solutions.

\subsection{The World Wide Web, Internet, And Networks}

Instant Messaging (IM): Instant messenger (IM) software can be downloaded from http://www.aol.com. This software allows students to instantly communicate amongst themselves and the instructor. 
Text Chat: A text chat capability is available at a number of Web servers to include http://chat.yahoo.com. The instructor can create either a public or a private chat room. A private chat room can be restricted to only invited members (such as students). The text chat applications typically require that the browser be capable of running Java applets. Some firewalls may not allow Java applets to pass through the firewall for security reasons.

Interactive Web Games: Students can interact over the Web playing such games as Backgammon or checkers at http://games.yahoo.com. Web games generally require that the browser be capable of running Java applets, which may have trouble passing through organization firewalls.

Audio chat: Audio chat can be conducted on a chat server by logging on at http://chat.yahoo.com and clicking on voice chat. The system will require both a microphone and a speaker (or headset) to use the function.

Web Page and Web Site Development: There are numerous free tools available for the creation and maintenance of web pages and web sites. An easy-to-use set of Web-based tools, along with free Web hosting, is available at http://www.geocities.com.

File Transfer Protocol (FTP) Software: An intuitive FTP client is available for download from http://www.ipswitch.com/downloads/. The FTP client allows the students to practice uploading and downloading files over the Internet using the FTP protocol. It should be noted that the Internet Explorer Web browser, as well as the Windows command prompt, also support the use of the FTP protocol, but are not as easy or intuitive as the FTP client software. It can be valuable to compare and contrast the different FTP tools.

Voice Over Internet Protocol (VOIP - Internet telephony): A VOIP application can be downloaded from http://www.net2phone.com. Using a headset with a microphone, students can make calls from their PCs to other PCs for free. It is also possible to make calls from a PC to a telephone for a nominal per minute charge.

Network Information: The Windows operating system has several native tools that can be used to review network settings and operations. By running the Windows Command Prompt (using Start, Run, 'command.com'), students have access to the following text based commands that return network-related information. The 'ipconfig /all' command lists the IP address and physical address of their PC. The 'tracert' command provides routing information between the student's PC and the Internet server that they are accessing. For example, 'tracert www.yahoo.com' provides routing hop information from the client to the Yahoo web server.

Domain Name Registrant Information: Students can review information related to who owns what domain names on the Internet using the Web form at http://www.networksolutions.com/en_US/whois/index.jhtml.

Web Facsimile Servers: The transmission and receipt of facsimile services over the web and email can be demonstrated using a free service at http://www.efax.com. An instructor can send a fax over a standard facsimile and demonstrate how it shows up in an email from the web fax server. However, when sending from a facsimile machine, it may be necessary to dial a long distance number to access the fax server. Alternatively, an instructor can demonstrate the sending of a facsimile from an email account to a facsimile machine.

\subsection{Electronic Commerce Resources}

Web Stores Tour: A virtual web store animated tour (with narration) is available at http://smallbusiness.yahoo.com/merchant/tour.php. It provides a tour of how a web store can be set up and used. Yahoo advertising is included as part of the tour.

Virtual Clothing Model Web Application: Students can create a 3-D model of themselves, then try items and outfits on their model to see how they would look on them. It is available at http://www.landsend.com.

Personal Shopper Web Application: A "personal shopper" suggests items and outfits that best suit a student's unique taste, style and preferences based on their inputs. It is available at http://www.landsend.com. 
Cookies and Cached Web Pages: Students can view cookies from their Web browser using the following menu navigation stream: Tools, Internet Options, General Tab, Settings Button, View Files. They can also view cached Web pages at this location.

Digital Certificates: Digital certificates for servers can be demonstrated by accessing a Web server with a digital certificate (such as the checkout, or shopping cart, for Web stores), and then clicking on the gold lock icon at the lower right of the Internet Explorer browser. An animated demonstration of how this is done is available at http://www.verisign.com/products/site/faq/index.html.

JavaScript and Java Applet Web Applications: JavaScript and Java applets can provide software functionality in Web browsers. Students can interact with online JavaScript calculators at http://www.webwinder.com/ wwhtmbin/javacalc.html\#stm12. Students can also review the JavaScript source code (by using the browser View, Source menu). A variety of Java applet based applications can be run from http://javaboutique.internet.com/ applets/. The Java applet source code is also available for download at this site.

eXtensible Markup Language (XML) Interactive Demonstration: An interactive demonstration of how XML can be used to query an inventory database is available at http://wsi.try.sybase.com/ebXMLSample/.

E-Commerce Interactive Animation: An interactive, animated flash demonstration on how online shopping works is available at http://www.learnthenet.com/english/animate/shopping.swf.

\subsection{Systems Development}

Project Management Software: A demonstration version of the FastTrack project management software can be downloaded from http://www.aecsoft.com/downloads/demo. The demonstration version is limited in functionality, but is probably sufficient for student familiarization exercises.

Integration Definition Function Modeling (IDEF): IDEF is a standard methodology for graphically representing decision, actions, and activities of an organization or system. It is used frequently for modeling business processes for business process reengineering and for modeling information systems. Software to develop IDEF diagrams is available at http://www.kbsi.com.

\subsection{It Infrastructures}

Peer-to-Peer File Sharing Software: Peer-to-peer file sharing software is available from a number of sources including http://www.kazaa.com. Students can use this software to share files between their client PCs. It may be necessary to control the usage of these peer-to-peer filesharing applications to limit the inappropriate downloading of copyrighted material, such as MP3 music files.

\subsection{It Security And Ethics}

Network Monitoring: Network monitoring software (commonly referred to as packet sniffers) can be downloaded from http://sumitbirla.com/network-spy/netspy.php. The packet sniffer can capture up to 3 minutes of packets from the local area network. The instructor can demonstrate the capture of information packets off the network. In order to protect the privacy of other users on the network, users should only capture packets traveling to and from their own computer.

Firewall: Firewall software can be downloaded from http://www.zonelabs.com. The firewall can be configured to offer various levels of protection. Using the alert function, users can monitor PC applications attempting to access the Internet as well as applications attempting to connect to their PCs in real time. Additionally, by examining the logs associated with the firewall, users can review all the traffic and attempted traffic between their PC and outside computers. 
Keystroke Logger Software: Keystroke logger software can record each of the keystrokes on a computer keyboard. Additionally, such software can also record screenshots of what is on the screen. It is possible to abuse this type of software to invade the privacy of other PC users, so the usage of this software should be closely controlled. A thorough review of computer ethics might be useful prior to introducing students to these types of tools. Keystroke logger software can be downloaded from http://www.bysoft.se/sureshot/.

Web-Based Port Scanning: Students can visit the Web page at http://grc.com/default.htm to initiate a security scan of their PC for open ports and shared files. This exercise can inform them of potential security holes in their PC.

Virus Scanner Software: Virus scanning software is available for download from http://www.avast.com/ i_idt_153.html.

Email Encryption Software: Students can encrypt emails and send them to email accounts with, and without, the necessary encryption keys. Email accounts that cannot decrypt the encrypted emails will see garbled, indecipherable text. Email accounts with the appropriate software and encryption keys will be able to decrypt the email messages and read them. A fairly common email encryption system, called PGP (pretty good privacy), is available for download at http://www.pgp.com/products/freeware.html.

Web Encryption Interactive Animation: An animated flash demonstration of how Web encryption works is available at http://www.learnthenet.com/english/animate/encrypt.htm.

File Encryption: Students can encrypt files to make them unintelligible to anyone but those people that know the key to decrypt the file. A software application to encrypt and decrypt files is available for download from http://kremlinencrypt.com/kremlin/pc_index.html.

Spyware Scanner: In many cases, unbeknownst to users, spyware is installed on their computers when they download and install other software. These spyware programs can violate user privacy by surreptitiously sending user information (such as browsing habits) over the Internet. A spyware detection and removal program is available at http://spybot.safer-networking.de/.

Web Privacy Test: Students can access a privacy test on a Web page that will inform them of the information that a Web site is able to glean about them, such as their IP address, PC name, contents of the clipboard, and browser version. The privacy test is available at http://www.anonymizer.com/privacytest/.

\subsection{Emerging It Trends And Technologies}

Interactive Three Dimensional (3D) Documentation: Students can interact with a 3D object embedded in a word processing document by downloading and installing the software available at http://www.ngrain.com/downloads/ register_no_mobilizer.html.

Cellular Phone Text, Image, and Sound Messaging: Users can send text messages from a Web site to a cellular phone number by using a Web form at http://www.vtext.com/customer_site/jsp/messaging_lo.jsp. There may be a nominal per message charge if the cellular phone user does not have unlimited text messaging as part of their cellular phone service. Thus, it might make sense to have students send text messages to a cellular phone number that does not charge by the message. An animated demonstration showing how to send images and sounds along with text messages from a PC to a cell phone is available at http://www.verizonwireless.com/ multimedia/pixplacedemo/pixhome.htm.

\subsection{Summary And Conclusion}

This paper has described a variety of free, interactive multimedia technologies that can support hands-on active learning in an MIS curriculum. A subset of these resources may be relevant to other college courses. 


\subsection{References}

1. Haag. S., Cummings, M., \& McCubbrey, D. (2003). Management Information Systems for the Information Age; New York: McGraw-Hill Irwin.

2. Harton, H., Richardson, D., Barreras, R. Rockloff, M., \& Latane, B. (2002). Focused interactive learning: A tool for active class discussion. Teaching of Psychology, 29, (1), 10-15.

3. Towson University. (2003). Towson University 2003-2004 Catalog.

Notes 


\section{Notes}

